\title{
Étude de la croissance diamétrique de quatre essences de bois d'œuvre exploitées à l'Est du Cameroun
}

\author{
Yao Lambert KOUADI01 ${ }^{*}$, Danho Fursy Rodelec NEUBA ${ }^{1}$, Moussa KONÉ ${ }^{1}$, Nils BOURLAND² et Jean- \\ Louis DOUCET² \\ 1 Unité de Formation et de Recherche en Sciences de la Nature (UFR, SN), Université Nangui Abrogoua, 02 BP 801 \\ Abidjan 02, Côte d'Ivoire \\ 2 Université de Liège, Gembloux Agro-Bio Tech, Unité de Gestion des Ressources Forestières et des Milieux \\ Naturels, Laboratoire de Foresterie des Régions Tropicales et Subtropicales, Passage des déportés 2, B-5030, \\ Gembloux, Belgique \\ Auteur correspondant email : lambertio10@yahoo.fr
}

Original submitted in on 20th January 2014. Published online at www.m.elewa.org on $31^{\text {st }}$ May 2014. http://dx.doi.org/10.4314/jab.v77i1.6

\section{RESUME}

Objectifs: Cet article étudie la croissance diamétrique de 4 essences commerciales à régénération naturelle déficiente exploitées à l'Est du Cameroun. II s'agit de Erythrophleum suaveolens (tali), Mansonia altissima (bété), Pericopsis elata (assaméla) et de Triplochiton scleroxylon (ayous).

Méthodologie et résultats : A cet effet, des dispositifs permanents de suivis de la croissance constitués par des circuits permanents ont été installés. Après 3 ans de mesures annuelles du diamètre des arbres, il a été obtenu un accroissement diamétrique moyen périodique de $0,68 \mathrm{~cm} /$ an pour $E$. suaveolens, de 0,37 $\mathrm{cm} /$ an pour $M$. altissima, de $0,32 \mathrm{~cm} /$ an pour $P$. elata et de $0,70 \mathrm{~cm} / a n$ pour $T$. scleroxylon. En considérant les accroissements périodiques moyens des deux classes de diamètre en dessous du diamètre minimum d'exploitation, des nouveaux taux de reconstitution pour les 4 essences ont été calculés.

Conclusions et application des résultats: Ces taux de reconstitution ont été comparés aux taux de reconstitution obtenus en utilisant les accroissements diamétriques fixés par l'administration camerounaise. On constate une surestimation des possibilités par l'administration pour $M$. altissima et $T$. scleroxylon et une sous-estimation des possibilités dans le cas de $E$. suaveolens. Pour $P$. elata, les taux de reconstitution obtenus dans nos dispositifs permanents sont quasi similaires à ceux calculés avec les accroissements diamétriques proposés par l'état Camerounais. Les résultats de cette étude pourront servir de référence à la gestion durable des essences à faible croissance diamétrique par un relèvement de leur diamètre minimum d'exploitation. Pour les essences à faible régénération naturelle comme celles prises en compte dans cette étude, des mesures d'intervention sylvicole devront être proposées.

Mots clés: essences commerciales, plan d'aménagement, accroissement diamétrique, dispositifs permanents, taux de reconstitution. 


\section{The study of the diametric growth of four timber species harvested in eastern Cameroon ABSTRACT}

Objectives: This article examines the diametric growth of 4 commercial species, with poor natural regeneration, exploited in eastern Cameroon. The species are Erythrophleum suaveolens (tali), Mansonia altissima (bété), Pericopsis elata (assaméla) and Triplochiton scleroxylon (ayous).

Methodology and results: For this purpose, permanent monitoring growth devices constituted by permanent circuits were installed. After 3 years of annual measurements of the tree diameter, a periodic diametric medium growth of $0.68 \mathrm{~cm} /$ year for $E$. suaveolens, $0.37 \mathrm{~cm} /$ year for $M$. altissima, $0.32 \mathrm{~cm} /$ year for $P$. elata and $0.70 \mathrm{~cm} /$ year for $T$. scleroxylon was obtained. Considering the periodic medium growth of two diameter classes below the minimum diameter for logging, a new replenishment rate for the 4 species was calculated.

Conclusion and application of findings:These replenishment rates were compared to the replenishment rate obtained using the diametric growth set by the Cameroonian government. There is an overestimation of the possibilities by the administration for $M$. altissima and $T$. scleroxylon and an underestimation of the possibilities in the case of $E$. suaveolens. For $P$. elata, the replenishment rate obtained in this study permanent devices is substantially similar to those calculated with the diametric growth proposed by the Cameroonian state. The results of this study will serve as a reference to the sustainable management of species with low diametric growth by raising their minimum logging diameter. For species with low natural regeneration as those considered in this study, silvicultural measures should be proposed.

Keywords: commercial timbers, management plan, diametric growth, permanent devices, replenishment rate.

\section{INTRODUCTION}

En 1992, le sommet de Rio a recommandé l'aménagement des forêts denses naturelles destinées à l'exploitation afin de les préserver sur le long terme (Catinot, 1999). Sur cette base et dans le souci de continuer à répondre aux besoins des populations locales ainsi que du marché des bois tropicaux toujours plus exigeant, le gouvernement camerounais a instauré une nouvelle législation forestière en 1994. Celle-ci impose aux sociétés d'exploitation forestière détentrices d'UFA (Unité Forestière d'Aménagement) de produire un plan d'aménagement puis, après validation par les services forestiers, de le mettre en application. La conception du plan d'aménagement repose sur des inventaires par échantillonnages statistiques des ressources ligneuses qualifiés d' « inventaires d'aménagement » (Forni, 1997 ; Dupuy et Forni, 1997). Ces inventaires sont réalisés à un taux de sondage supérieur ou égal à $1 \%$ pour une concession de superficie inférieure à 50.000 ha ou d'au moins 0,5\% pour une concession de superficie supérieure ou égale à 50.000 ha
(Kouadio, 2009). Les résultats de l'inventaire permettent de dresser la liste des essences sur lesquelles seront orientées les investigations (Fargeot et al., 2004). Ainsi, après avoir établi les structures de population et calculé les taux de reconstitution des essences exploitables, il sera déterminé les paramètres d'aménagement (rotation, diamètres minima d'exploitation, etc.) compatibles avec le renouvellement de la ressource (Doucet, 2003; Kouadio, 2003). Reposant principalement sur le calcul des possibilités ou de reconstitution des essences commerciales, le plan d'aménagement n'est basé que sur le renouvellement des essences au cours d'une rotation de 30 ans (Gayot et Sist, 2004). L'aménagement portera sur un nombre limité d'espèces de qualité technologique reconnue, relativement abondantes et déjà appréciées sur le marché. Ainsi, pour les essences présentant un taux de reconstitution insuffisant entre deux coupes, le plan d'aménagement peut proposer une augmentation du diamètre minimum d'exploitation (DME) afin de retrouver un volume acceptable lors 
de la prochaine rotation. Toutefois, les simulations sur le plus long terme (2 rotations) sont généralement inexistantes et nullement exigées par la législation. Cela ne peut qu'hypothéquer le maintien d'espèces à régénération naturelle déficiente comme Alstonia boonei, Erythrophleum suaveolens, Mansonia altissima, Pericopsis elata, Terminalia superba et Triplochiton scleroxylon, (Doucet, 2003 ; Kouadio, 2003 et 2009 ; Bourland et al., 2012a). Par ailleurs, le calcul des possibilités se fonde sur trois paramètres essentiels de la dynamique : l'accroissement annuel du diamètre, la mortalité naturelle annuelle et les dégâts d'exploitation (Bayol et Borie, 2004). Or, ces paramètres de la dynamique des populations restent insuffisamment connus. Faute de valeurs estimées localement, des données moyennes issues de la littérature sont fréquemment utilisées (Fétéké et al., 2003; 2004). L'origine de ces données demeure souvent méconnue. $\mathrm{Ne}$ reposant pas toujours sur des études scientifiques fiables, elles peuvent biaiser considérablement les estimations des taux de reconstitution. Dans le meilleur des cas, les données spécifiques aux espèces sont considérées. Par exemple, le taux de mortalité est toujours fixé à $1 \%$, ses effets se cumulent sur toute la durée de la rotation. II n'y a aucune garantie que les conditions locales dans

\section{MATERIEL ET METHODES}

Site d'étude: L'étude s'est déroulée dans la concession forestière attribuée à la société Pallisco, en République du Cameroun, dans la province de l'Est, district du Dja, village de Mindourou (Figure 1). La zone d'étude est située entre les latitudes $3^{\circ} 10^{\prime}$ et $3^{\circ} 44^{\prime}$ Nord et entre les longitudes $14^{\circ} 05^{\prime}$ et $14^{\circ} 31^{\prime}$ Est, sur une superficie de 341708 ha. Les précipitations s'élèvent en moyenne à $1650 \mathrm{~mm}$ par an et sont réparties en quatre périodes: une grande saison des pluies (d'août à novembre), une saison sèche très marquée (de décembre à février), une petite saison des pluies (de mars à juin) et une petite saison sèche (juillet) dont l'ampleur est très variable d'une année à l'autre. La température moyenne est de $24^{\circ} \mathrm{C}$. Le relief est doux et assez uniforme. Les dépressions sont faibles mais bien marquées. Le réseau hydrographique dessine un maillage régulier de petites vallées à fond plat et marécageux. L'altitude varie de 540 à $806 \mathrm{~m}$. lesquelles ces paramètres ont été estimés correspondent à celles du peuplement étudié. Selon Delegue et al. (1998), la dynamique forestière varie avec le temps, avec la classe de diamètre et avec le statut « social» des individus, les arbres dominés poussant moins vite. Des études basées sur des simulations à l'aide de modèles de dynamique forestière montrent qu'une mauvaise estimation des paramètres de la dynamique forestière peut conduire à des biais importants dans l'estimation des possibilités (Picard et Gourlet-Fleury, 2007). Une meilleure estimation des paramètres de la dynamique forestière est donc nécessaire pour améliorer la fiabilité du calcul de la possibilité et par la même occasion des plans d'aménagement

Dans cet article, nous avons cherché à évaluer la dynamique des populations de 4 essences forestières à régénération naturelle déficiente. II s'agit de Ertrophleum suaveolens, Mansonia altissima, Pericopsis elata et de Triplochiton scleroxylon. A cette fin, nous avons déterminé la croissance diamétrique de ces 4 essences dans des dispositifs permanents de suivi de la croissance installés en périphérie et dans une concession forestière située à l'Est du Cameroun dans le département d'Abong Mbang, district du Dja, dans le village de Mindourou.

La plus grande partie de la zone d'étude repose sur le complexe de base du précambrien inférieur dont les principales roches sont les micaschistes gris sombres argentés. Du point de vue pédologique, les sols rencontrés dérivent de roches métamorphiques. Sur la terre ferme, ce sont principalement des sols ferralitiques rouges ou jaunes typiques. Ils sont en général profonds, argileux, meubles, perméables et présentent souvent peu d'humus. Leur teneur en bases échangeables est faible. Ce sont des sols pauvres, acides et fragiles. On y rencontre une forêt de transition entre les types sempervirents et semi-sempervirents. Elle se caractérise par la rareté des Caesalpiniaceae, et la présence de nombreuses Euphorbiaceae et Olacaceae. Parmi les arbres de grande taille, les Meliaceae ainsi que diverses Sterculiaceae et Ulmaceae sont bien représentées (Letouzey, 1968). 


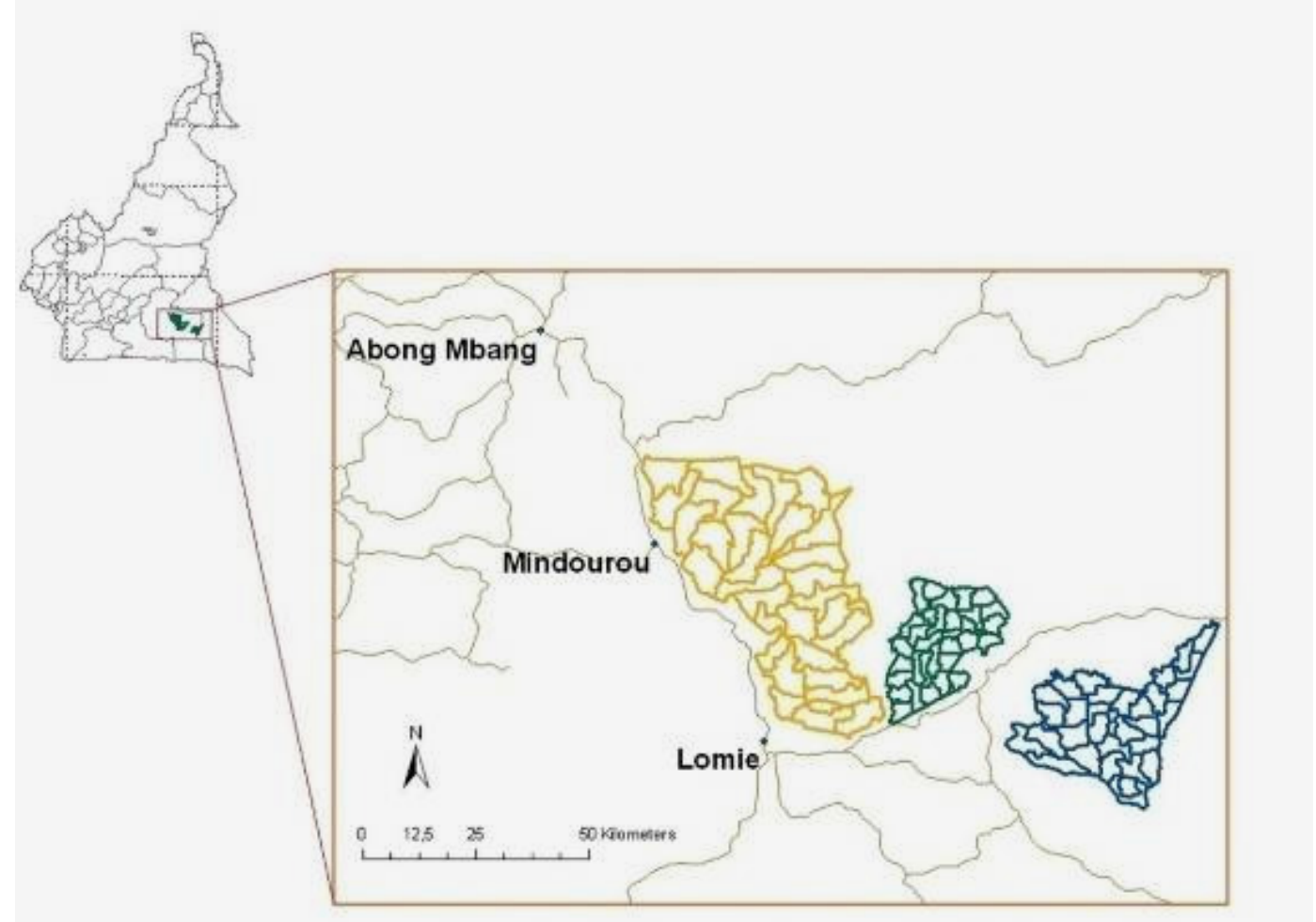

Figure 1 : Localisation de la zone d'étude (341.708 ha)

Dispositif d'étude: Afin d'étudier les paramètres démographiques pouvant affecter les structures des populations de quatre essences, des dispositifs permanents de suivi de la croissance ont été installés en périphérie et dans la concession forestière de la société Pallisco. Pour chaque essence, le dispositif implanté consiste en un circuit (piste) reliant les différents pieds comme indiqué par la figure 2. La hauteur optimale de mesure du diamètre a été définie pour chaque arbre du circuit par convention à $1,30 \mathrm{~m}$ au dessus du niveau du sol.

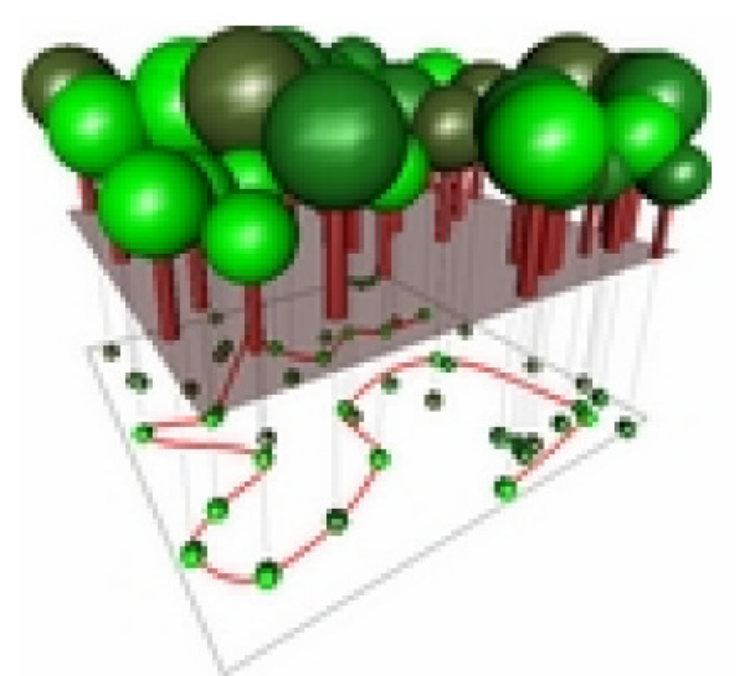

Figure 2 : Mise en évidence d'un dispositif permanent constitué par un circuit (Picard et Gourlet-Fleury, 2007) 


\section{Kouadio et al. J. Appl. Biosci. 2014. Étude de la croissance diamétrique de quatre essences de bois d'œuvre exploitées a l'est du Cameroun}

Toutefois, T. scleroxylon étant munis de contreforts, les mesures ont été rendues impossibles à une telle hauteur. Dans un tel cas, il convient d'effectuer la mesure au moins $50 \mathrm{~cm}$ au-dessus de tout contrefort ou déformation. La zone de mesure a ensuite été soigneusement nettoyée. Les écailles et les épiphytes ont été enlevés car elles risqueraient de générer des biais, y compris des accroissements négatifs si elles venaient à disparaître entre deux mesures. Après le nettoyage, un ruban adhésif a été posé sur la zone de mesure. Le diamètre a alors été mesuré à l'aide d'un ruban métrique adéquat, correctement tendu. Ensuite, après enlèvement du ruban métrique, les zones bordant le ruban adhésif ont été mises en peinture afin qu'en retirant celui-ci, l'on dispose d'une matérialisation nette du pourtour de remesurage. Ces zones ont été repeintes à chaque passage afin que la matérialisation persiste durant toute la période d'étude (figure 3).

Année 0 : Installation du dispositif, pose d'un ruban adhésif, mesure du diamètre sur le ruban posé, badigeonnage du pourtour de l'arbre de part et d'autre du ruban adhésif

Année 0 (suite)

Retrait du ruban adhésif : matérialisation de la ligne circulaire représentant le niveau de mesure et garantissant sa répétabilité dans le temps

Année 1 et suivantes : pose du ruban adhésif sur le niveau de mesure, remesurage sur le ruban, remise en peinture et retrait du ruban
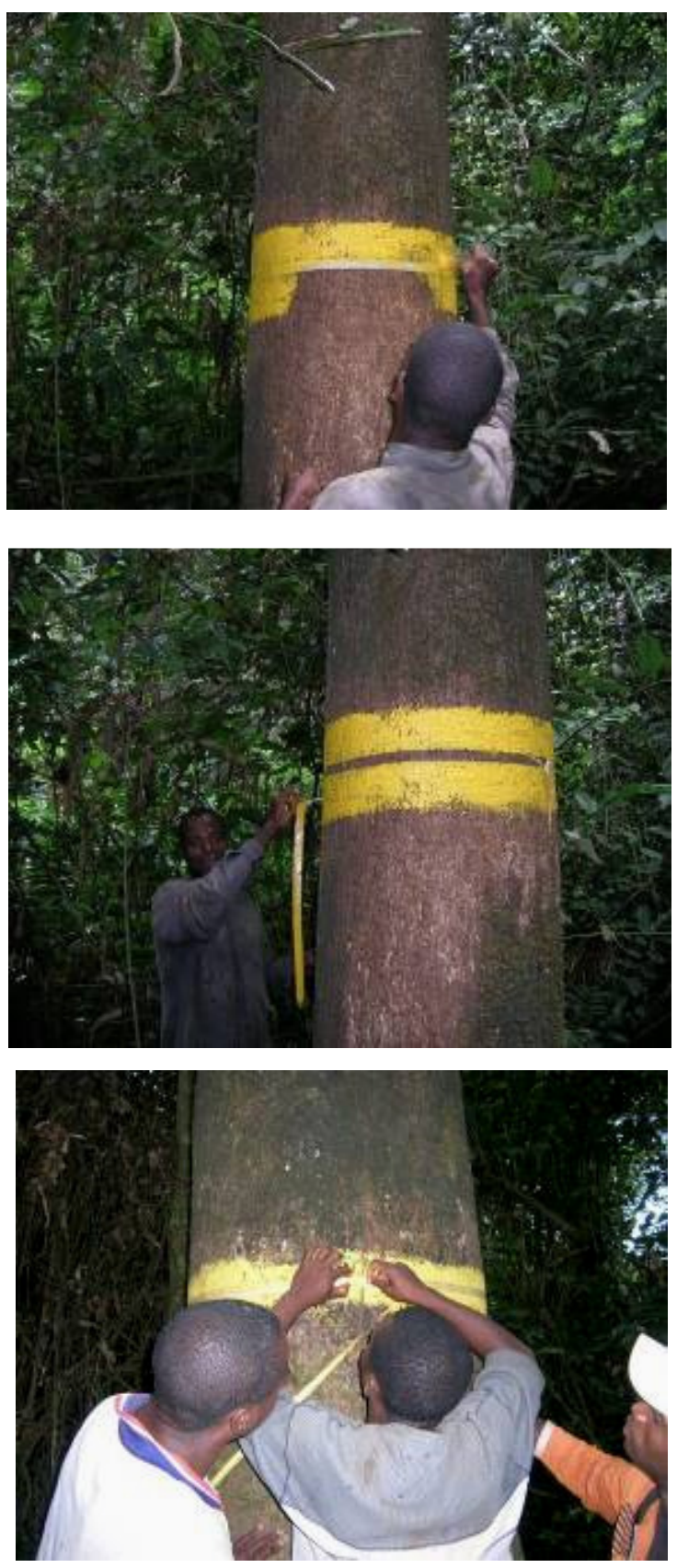

Figure 3 : Procédure de mesure et de remesurage du diamètre d'un arbre (Doucet et al., 2007) 
L'accroissement diamétrque annuel a été obtenu en réalisant la différence entre deux mesures successives. Les remesurages ont été effectués tous les ans pendant trois années. Afin d'étudier l'influence du statut social des arbres sur leur croissance diamétrique, ces derniers ont été classés dans trois catégories (figure 4) :

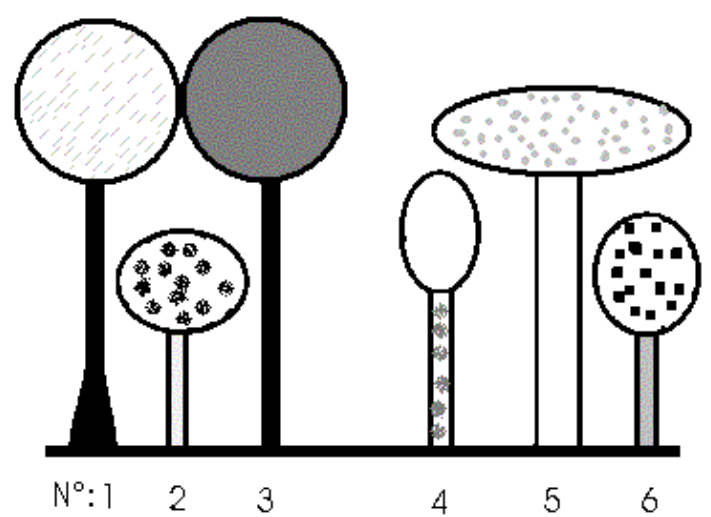

Figure 4 : Évaluation du statut social d'un arbre: Arbre dominant: $n^{\circ} 5$; arbres codominants $n^{\circ} 1$ et 3 ; arbres dominés : $n^{\circ} 2,4$ et 6

- les arbres dominants dont les cimes sont émergentes dans la canopée ;

- les arbres codominants, c'est-à-dire ceux dont les cimes sont situées dans le même étage que leurs voisins ;
- les arbres dominés, correspondent à ceux dont les cimes sont surpassées par celles de leurs voisins.

- Le tableau 1 montre pour chaque essence, le nombre d'individu recensé.

Tableau 1 : Nombre de tiges par classe de diamètre dans les dispositifs permanents

\begin{tabular}{|c|c|c|c|c|}
\hline $\begin{array}{l}\text { Classes de } \\
\text { diamètre }\end{array}$ & $\begin{array}{l}\text { Pericopsis. } \\
\text { elata }\end{array}$ & Triplochiton scleroxylon & Mansonia altissima & Erytrophleum suaveolens \\
\hline 1 & 3 & 2 & 3 & \\
\hline 2 & 4 & 3 & 12 & \\
\hline 3 & 6 & 8 & 9 & 1 \\
\hline 4 & 10 & 8 & 16 & 2 \\
\hline 5 & 5 & 17 & 12 & 6 \\
\hline 6 & 10 & 22 & 3 & 10 \\
\hline 7 & 8 & 15 & & 9 \\
\hline 8 & 6 & 4 & & 3 \\
\hline 9 & 5 & 1 & & 5 \\
\hline 10 & 2 & & & 1 \\
\hline 11 & & & & 1 \\
\hline $\begin{array}{l}\text { Nombre de } \\
\text { tiqes }\end{array}$ & 59 & 80 & 55 & 38 \\
\hline
\end{tabular}

La classe 1 correspond à l'intervalle 10-19,9 cm ; la classe 2 : 20 à 29,9 cm; etc.

\section{RESULTATS}

Distribution des effectifs dans les différents circuits :Au sein des quatre dispositifs permanents, les populations affichent des distributions en cloche (figure 5). Un déficit de tiges est constaté en outre au niveau de $P$. elata dans la classe de diamètre $5(50-59,9 \mathrm{~cm})$, de $M$. altissima dans la classe $3(30-39,9 \mathrm{~cm})$ et de $E$. suaveolens dans la classe $8(80-89,9 \mathrm{~cm})$. Par ailleurs, très peu de classes de diamètre ont été recensées pour au niveau de M. altissima soit 6 classes alors qu'au niveau de $E$. suaveoelens et de $P$. elata, de très 


\section{Kouadio et al. J. Appl. Biosci. 2014. Étude de la croissance diamétrique de quatre essences de bois d'œuvre exploitées a l'est du Cameroun}

grosses tiges de diamètre $\geq 100 \mathrm{~cm}$ ont été inventoriées. M. altissima est en effet considérée comme une espèce n'atteignant jamais d'importants diamètres, contrairement aux trois autres essences. De plus, aucun individu appartenant aux classes de diamètre 1 et 2 n'a été inventorié chez $E$. suaveolens.
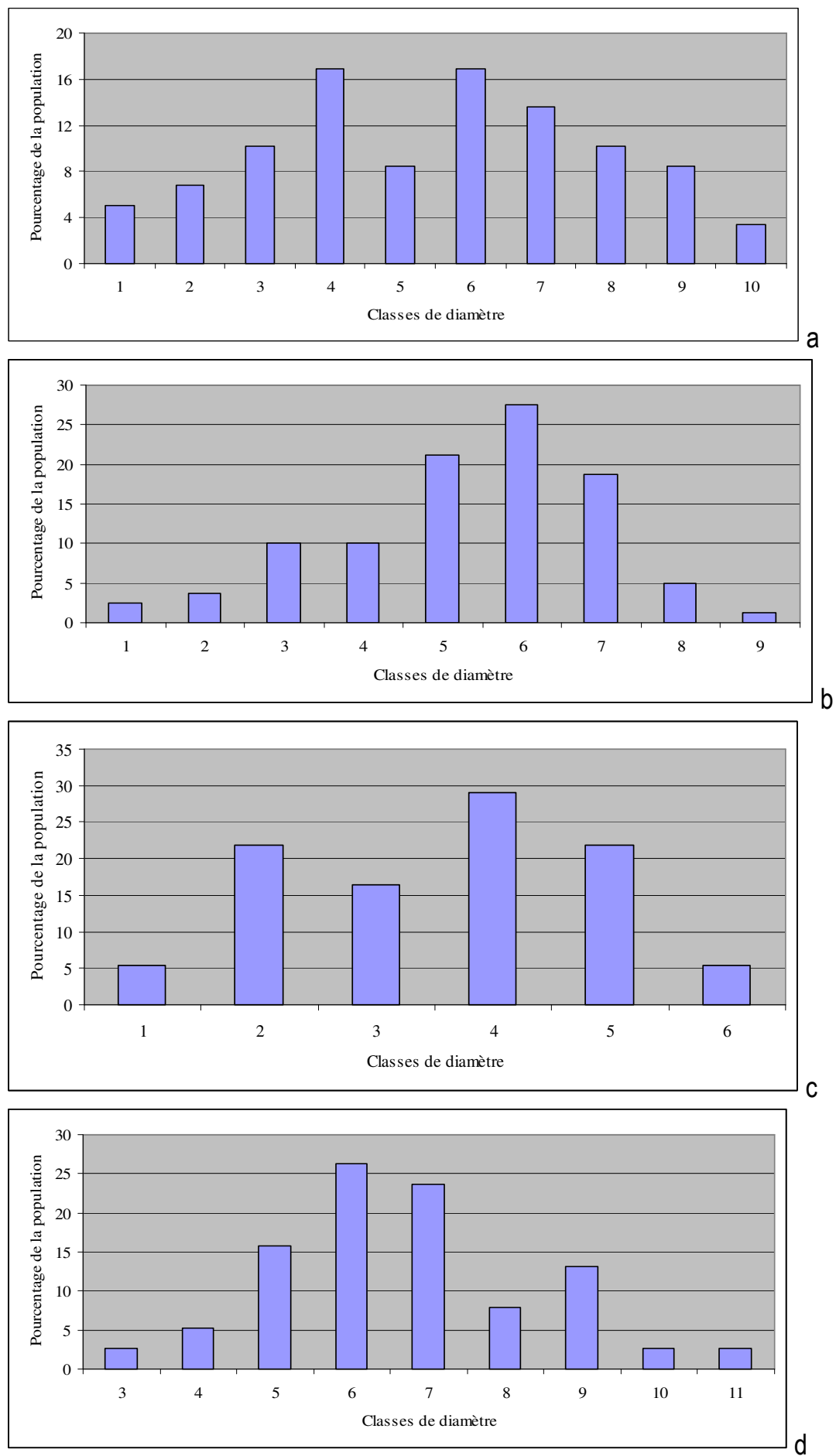

Figure 5 : Distribution diamétrique des tiges inventoriées dans les dispositifs permanents d'étude de la croissance. Classe $1=10-19,9 \mathrm{~cm}$, classe $2=20-29,9 \mathrm{~cm}$, classe $3=30-39,9 \mathrm{~cm}$, etc. a. P. elata ; b. T. scleroxylon; c. $M$. altissima; d. E. suveolens. 


\section{Kouadio et al. J. Appl. Biosci. 2014. Étude de la croissance diamétrique de quatre essences de bois d'œuvre exploitées a l'est du Cameroun}

Accroissements diamétriques : La figure 6 présente les accroissements diamétriques annuels moyens par classe de diamètre des 4 essences soumises à notre étude. Certaines classes de diamètre ont été regroupées car très peu représentées. Pour chaque arbre, l'accroissement diamétrique correspond à la soustraction du diamètre mesuré après 3 ans de celui mesuré au début de cette étude.

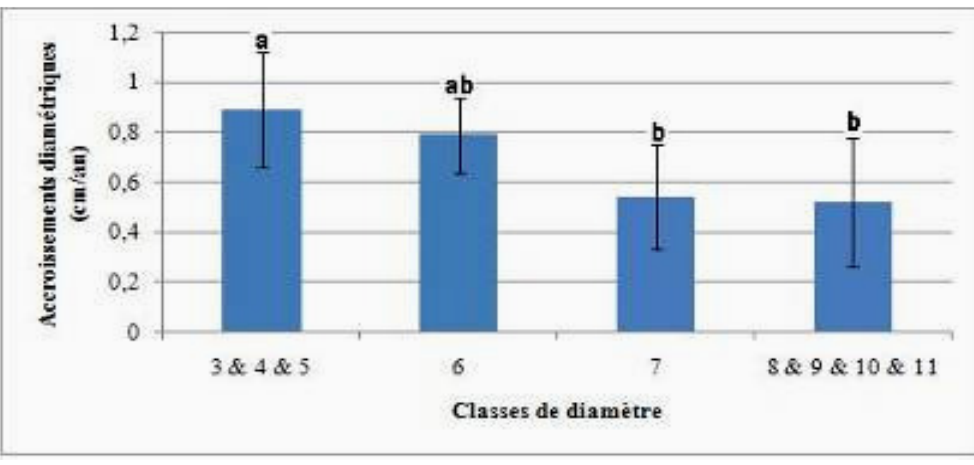

a- E. suaveolens

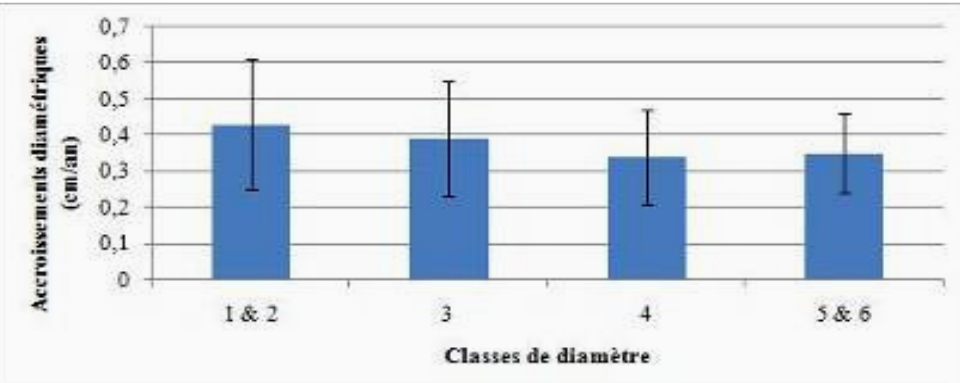

b- M. altissima

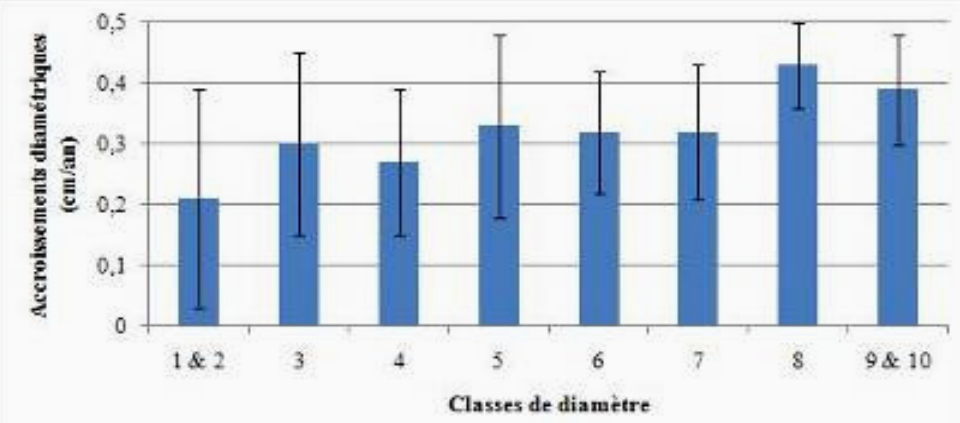

c- P. elata

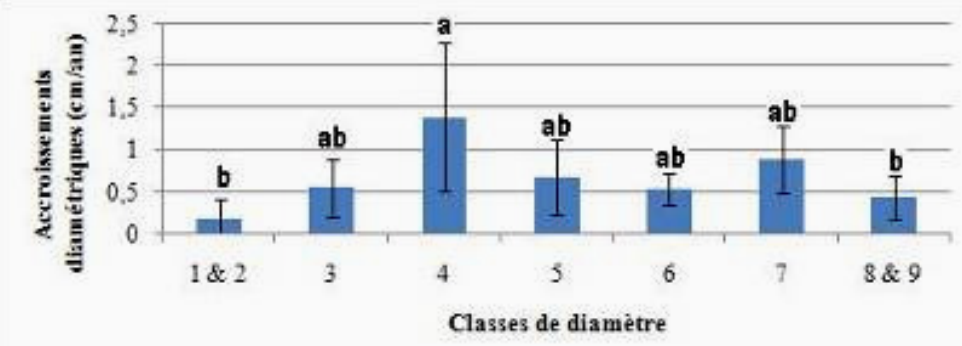

d- T. scleroxylon

Figure 6 : Évolution des accroissements diamétriques annuels moyens ( \pm intervalles de confiances à $95 \%$ audessus des histogrammes) en fonction des classes de diamètre de $E$. suaveolens, $M$. altissima, $P$. elata et $T$. scleroxylon; Classe $1=10-19,9 \mathrm{~cm}$, classe $2=20-29,9 \mathrm{~cm}$, classe $3=30-39,9 \mathrm{~cm}$, etc., les moyennes d'accroissements diamétriques des classes de diamètre marquées de la même lettre ne sont pas différentes entre elles 


\section{Kouadio et al. J. Appl. Biosci. 2014. Étude de la croissance diamétrique de quatre essences de bois d'œuvre exploitées a l'est du Cameroun}

L'accroissement diamétrique annuel moyen obtenu après cette période est de $0,32 \pm 0,04 \mathrm{~cm} /$ an pour $P$. elata. On observe au niveau de cette essence une tendance légèrement croissante dans le temps des accroissements diamétriques jusqu'à la classe de diamètre $8(80-89,9 \mathrm{~cm})$ suivie d'une baisse. Aucune différence significative n'a été mise en évidence entre les accroissements diamétriques moyens en fonction des classes de diamètre (ANOVA; $D L=7 ; F=1,52 ; p$ $=0,180)$. Les tiges de la classe de diamètre 9 à 10 susceptibles de dépasser le DME $(100 \mathrm{~cm}$ dbh) après une rotation ont un accroissement moyen périodique de $0,39 \pm 0,09 \mathrm{~cm} / \mathrm{an}$. L'accroissement diamétrique moyen de $T$. scleroxylon, tous diamètres confondus, est de $0,70 \pm 0,15 \mathrm{~cm} / \mathrm{an}$. La distribution par classe des accroissements diamétriques est traduite par une courbe en cloche avec un pic d'accroissement de 1,39 $\pm 0,89 \mathrm{~cm} /$ an dans la classe de diamètre 4 . Les tiges des classes de diamètre 6 et 7 potentiellement exploitables à la prochaine rotation (DME $=80 \mathrm{~cm} \mathrm{dbh}$ ) présentent un accroissement diamétrique moyen périodique de 0,68 $\pm 0,18 \mathrm{~cm} / \mathrm{an}$. L'analyse de la variance montre l'existence d'une différence significative entre les accroissements diamétriques moyens obtenus pour chaque classe de diamètre (ANOVA, $F=2,58 ; D L=6 ;{ }^{*} p=0,025$ ). Les accroissements diamétriques moyens sont significativement différents entre les classes de diamètre 1 et 2 regroupées et la classe 4 et entre la classe de diamètre 4 et les classes 8 et 9 également regroupées. En ce qui concerne $M$. altissima, l'accroissement diamétrique annuel moyen après trois années de mesure est de 0,37 $\pm 0,07 \mathrm{~cm} / \mathrm{an}$. L'accroissement diamétrique adopte une légère tendance à la baisse au cours de la vie de cette essence, observation confirmée par la figure 7 . Les tiges des classes de diamètre 5 à 6 , c'est-à-dire les arbres qui constitueront probablement le stock exploitable lors de la prochaine exploitation pour un DME de $60 \mathrm{~cm}$, donnent un accroissement diamétrique de $0,35 \pm 0,11 \mathrm{~cm} / a n$. II n'y a cependant pas de différence significative entre les accroissements moyens des différentes classes de diamètre (ANOVA; $F=0,36 ; D L=3 ; p=0,781)$. Enfin, après trois années de mesure, un accroissement diamétrique annuel moyen de $0,68 \pm 0,10 \mathrm{~cm} / \mathrm{an}$ a été observé chez $E$. suaveolens. L'accroissement diamétrique diffère significativement d'une classe de diamètre à l'autre (ANOVA, DL $=3 ; \mathrm{F}=3,64 ;{ }^{*} \mathrm{p}=0,022$ ). Aussi, l'accroissement diamétrique de cette essence adopte til une tendance baissière dans le temps. Les tiges des classes de diamètre 3 à 5 (DME $=50 \mathrm{~cm}$ dhp) qui sont susceptibles d'être exploitées à la prochaine rotation présentent un accroissement diamétrique de 0,89 \pm $0,23 \mathrm{~cm} / \mathrm{an}$

Influence du statut sociale des arbres sur les accroissements diamétriques : Le tableau 2 présente les accroissements diamétriques des 4 essences en fonction du statut social des arbres dans la canopée. Chez $P$. elata, les moyennes des accroissements diamétriques sur les trois années de mesure montrent les plus faibles valeurs au niveau des arbres dominés et codominants. Cette constatation est confirmée par les résultats de l'analyse de la variance. Celle-ci montre une différence significative entre les accroissements moyens et le statut des arbres (ANOVA de KruskalWallis, $H=7,845 ;{ }^{*} p=0,019$ ). Cette influence du statut social sur l'accroissement diamétrique ne concerne pas T. scleroxylon (ANOVA, $\mathrm{F}=0,317 ; \mathrm{DL}=2, \mathrm{p}=0,728$ ), M. altissima (ANOVA; $F=1,47 ; D L=2 ; p=0,239$ ) et E. suaveolens (ANOVA, $F=2,80, D L=2, p=0,074$ ).

Tableau 2: Accroissements diamétriques ( \pm intervalles de confiance) en $\mathrm{cm} / \mathrm{an}$ en fonction du statut social des arbres dans la canopée.

\begin{tabular}{llll}
\hline & Dominants & Codominants & Dominés \\
\hline E. suaveolens & $0,55 \pm 0,13$ & $0,77 \pm 0,19$ & $0,66 \pm 0,39$ \\
M. altissima & $0,46 \pm 0,16$ & $0,32 \pm 0,08$ & $0,42 \pm 0,15$ \\
P. elata & $0,37 \pm 0,05$ & $0,34 \pm 0,09$ & $0,32 \pm 0,06$ \\
T. scleroxylon & $0,76 \pm 0,31$ & $0,69 \pm 0,20$ & $0,54 \pm 0,55$ \\
\hline
\end{tabular}




\section{DISCUSSION}

Les accroissements annuels moyens périodiques des classes de diamètre précédant les DME obtenus pour les quatre espèces peuvent être comparés à ceux proposés par l'état camerounais lors de la réalisation des plans d'aménagement (tableau 3). On observe que la valeur mesurée pour $E$. suaveolens $(0,89 \pm 0,23$ $\mathrm{cm} / \mathrm{an}$ ) est nettement plus élevée que celle prise en compte dans les plans d'aménagement qui est de 0,40 $\mathrm{cm} / \mathrm{an}$. Concernant $M$. altissima, $P$. elata et $T$. scleroxylon, les différences ne sont pas énormes. Toutefois, les accroissements diamétriques que nous avons mesurés sont légèrement inférieurs à ceux fournis par l'État dans les cas de M. altissima et de $T$. scleroxylon et quasi similaire dans le cas de $P$. elata. Ces différences, fussent-elles minimes, peuvent, sur une longue période de temps donnée (30 ans pour une rotation) avoir des influences sur les prévisions.

Tableau 3 : Comparaison des accroissements diamétriques fixés par l'administration et ceux issus des dispositifs permanents

\begin{tabular}{l|l|l}
\hline Espèces & $\begin{array}{l}\text { Accroissement diamétrique } \\
\text { fixé par l'administration } \\
\text { (cm/an) }\end{array}$ & $\begin{array}{l}\text { Accroissements diamétriques issus de } \\
\text { nos dispositifs permanents } \\
\text { (cm/an) }\end{array}$ \\
\hline Erythrophleum suaveolens & 0,4 & $0,89 \pm 0,23$ \\
Mansonia altissima & 0,5 & $0,35 \pm 0,11$ \\
Pericopsis elata & 0,4 & $0,39 \pm 0,09$ \\
Triplochiton scleroxylon & 0,9 & $0,68 \pm 0,18$ \\
\hline \multicolumn{2}{l}{ Les accroissements périodiques moyens des deux classes de diamètre en dessous du diamètre minimum } \\
d'exploitation ont été considérés
\end{tabular}

Une comparaison des taux de reconstitution calculés avec les accroissements diamétriques fixés par l'administration et ceux calculés à partir des résultats issus des dispositifs permanents montre deux cas de figure (tableau 4) :

- le premier est une surestimation des accroissements diamétriques par l'administration. Dans cette situation, les taux de reconstitution sont également surestimés. C'est le cas ici de M. altissima et de $T$. scleroxylon.

- le deuxième est une sous-estimation de l'accroissement diamétrique par l'administration comme pour $E$. suaveolens. Ici, le taux de reconstitution réel est plus élevé que les prévisions.

Tableau 4 : Comparaison des taux de reconstitution en pourcentage obtenus avec les accroissements diamétriques fixés par l'administration Camerounaise et ceux obtenus avec les accroissements diamétriques issus des dispositifs permanents sur deux massifs forestiers concédés à la société d'exploitation forestière Pallisco

\begin{tabular}{lccccc}
\hline Massifs forestiers & $\begin{array}{c}\text { Origine } \\
\text { accroissements } \\
\text { diamétriques }\end{array}$ & E. suaveolens & M. altissima & P. elata & T. scleroxylon \\
\hline UFA 10 030 et 10 & Fixé par l'État & 13,5 & 147,6 & 489,5 & \\
031 & Dispositifs & 27,5 & 104,6 & 468,5 & 25,5 \\
UFA 10 039 & Fixé par l'État & 7,9 & 0 & 117,2 & 26,7 \\
& Dispositifs & 17,3 & 0 & 114,3 & 23,8 \\
\hline
\end{tabular}

L'analyse des accroissements diamétriques en fonction des classes de diamètre montre l'existence de différences significatives chez $T$. scleroxylon. Pour $M$. altissima, on note une décroissance des accroissements diamétriques dans le temps. Celle-ci a déjà été mise en évidence par Durrieu de Madron
(2003). Pour les trois autres espèces, leur tempérament héliophile favorise une installation dans les peuplements ouverts (Doucet, 2003). Toutefois, il peut arriver que les tiges dominées ne grandissent pas ou très peu. Cela est particulièrement confirmé en ce qui concerne $P$. elata où les arbres d'un statut social 
dominant fournissent des accroissements diamétriques significativement supérieurs à ceux des tiges codominantes et dominées (Bourland et al., 2012b). Cependant, à partir d'un certain âge, commence un ralentissement du taux de production de matière

\section{CONCLUSION}

L'étude de la dynamique de $E$. suaveolens, de $M$. altissima, de $P$. elata et de $T$. scleroxylon a permis de déterminer les accroissements diamétriques annuels moyens périodiques de ces espèces, la connaissance de ces données étant fondamentale pour un aménagement efficace et une gestion durable de ces espèces vulnérables. Les accroissements diamétriques obtenus ont permis de proposer de nouvelles valeurs de reconstitution pour ces espèces. Ces valeurs sont plus proches de la réalité que les valeurs obtenues avec les accroissements diamétriques fixés par l'administration camerounaise. II aurait été judicieux d'établir un lien entre les accroissements diamétriques

\section{REMERCIEMENTS}

Toutes nos pensées vont à l'ASBL Nature + pour le financement de ce travail et à Loïc DOUAUD, directeur Général adjoint de la société d'exploitation forestière Pallisco pour avoir accepté que ce travail se déroule

\section{REFERENCES}

Bayol, N. \& Borie, J.-M. (2004) Itinéraires techniques d'aménagement des forêts de production en Afrique centrale. Bois et forêts des Tropiques 28(3), 35-49.

Bourland, N., Kouadio, Y. L., Fétéké, F., Lejeune, P., Doucet, J-L. (2012a) Ecology and management of Pericopsis elata (Harms) Meeuwen (Fabaceae) populations : a review Biotechnol. Agron. Soc. Environ.16(4), 486498

Bourland, N., Kouadio, Y. L., Lejeune, P, Sonké B., Philippart J. Daïnou K., Fétéké, F., Doucet, JL. (2012b) Ecology of Pericopsis elata(Fabaceae), an Endangered Timber Species in Southeastern Cameroon, BIOTROPICA 44(6): 840-847.

Brienen, J.W. \& Zuidema, P.A. (2006) Lifetime growth patterns and ages of Bolivian rain forest trees obtained by tree ring analysis. Journal of Ecology, 94, 481-493.

Catinot, R. (1999) La gestion durable des forêts tropicales : où en est-elle six ans après le ligneuse. Ce phénomène a été également observé par Brienen et Zuidema (2006) et King et al., (2006). Le nombre de tiges suivies est cependant trop faible dans cette étude pour conclure en ce qui concerne les espèces suivies.

et les précipitations pour savoir si les accroissements obtenus sont liées à la pluviométrie d'une année à l'autre. En outre, une relation devrait être établie entre les accroissements diamétriques et la fructification. Cette étude devra par ailleurs s'étendre à d'autres essences commerciales à régénération naturelle déficiente afin de proposer des mesures de gestion durable de ces essences problématiques. Ces mesures seront basées sur la mise en place de programmes d'intervention sylvicoles et le relèvement des diamètres minima d'exploitation pour les essences à faibles croissances diamétriques.

dans sa concession. Nous remercions également Théophile AYOL, Paul ZOK, Crépin DJOPANDE pour leur aide sur le terrain.

sommet de Rio ? Bois et Forêts des tropiques, 261(3), 61-69.

Delege, M.A., Fuhr, M., Nasi, R., \& Minkoué, J.M. (1998). Dynamique et croissance de l'okoumé en zone côtière du Gabon. Cirad-forêt, série Forafri, Montpellier, France, $55 \mathrm{p}$.

Doucet, J-L. (2003) L'alliance délicate de la gestion et de la biodiversité dans les forêts du Gabon. Thèse de doctorat, Faculté Universitaire des Sciences Agronomiques Gembloux, $323 \mathrm{p}$.

Dupuy, B. \& Forni, E. (1997) Aménagement forestiers à l'Est du Cameroun. Bois et Forêts des tropiques, 254, 39-50.

Durrieu De Madron, L. (2003) Accroissement diamétrique du bété et de l'iroko. Bois et Forêts des tropiques, 275, 83-87.

Fargeot, C., Forni, E., \& R., N. (2004) Réflexions sur l'aménagement des forêts de production du bassin du Congo. Bois et Forêts des tropiques, 281(3), 19-34.

Fétéké, F., Nkolong, E., \& Hubert, D. (2003). Plan d'aménégement de l'Unité Forestière d'Aménagement 10.039. Ets Assene Nkou, 
Cellule d'Aménagement R. Pallisco, Nature Plus, $166 \mathrm{p}$.

Fétéké, F., Nkolong, E., \& Hubert, D. (2004). Plan d'aménagement des Unités Forestières d'Aménagement $n^{\circ} 10$ 041, 10042 et 10044 regroupées, Cellule d'aménegement Pallisco, Nature Plus, $173 p$

Forni, E. (1997). Type de forêts dans l'Est du Cameroun et étude de la structure diamétrique de quelques essences. Mémoire de DEA, Faculté Universitaire des Sciences Agronomiques de Gembloux, $64 p$

Gayot, M. \& Sist, P. (2004) Vulnérabilité des espèces de maçanranduba face à l'exploitation en $\mathrm{A}$ mazonie brézilienne: nouvelles normes d'exploitation à définir Bois et Forêts des tropiques, 280 (2), 75-90.

King, D.A., Davies, S.J., \& Noor, N.S.M. (2006) Growth and mortality are related to adult tree size in a Malaysian mixed dipterocarp forest. Forest Ecology and Management, 223, 152-158.
Kouadio, Y.L. (2003). Étude des potentialités sylvicoles des peuplements d'okoumé (Aucoumea klaineana Pierre) dans le region de Mandji au Gabon. Mémoire de DEA, Faculté Universitaire des Sciences Agronomiques Gembloux, 79 p.

Kouadio, Y.L. (2009). Mesures sylvicoles en vue d'améliorer la gestion des populations d'essences forestières commerciales de l'Est du Cameroun, Thèse de doctorat, Faculté Universitaire des Sciences agronomiques de Gembloux, $271 \mathrm{p}$.

Letouzey, R. (1968) Étude phytogéographique du Cameroun. Editions Paul Lechevalier, Paris, $511 \mathrm{p}$.

Picard, N. \& Goulet-Fleury, S. (2007). Propositions de directives pour l'installation de parcelles permanentes. CIRAD Departement Environnement et Sociétés UPR Dynamique des forêts naturelles, Yaoundé, Cameroun, $220 \mathrm{p}$. 